\title{
Analyse des Déterminants des Emplois Stables en Côte d'Ivoire
}

\author{
N'Gratier Antoine, Docteur en Sciences Economiques
}

Enseignant-chercheur, UFR des Sciences Economiques et de Gestion

Université Félix Houphouët Boigny d'Abidjan, Côte d'Ivoire

Doi:10.19044/esj.2019.v15n25p262 URL:http://dx.doi.org/10.19044/esj.2019.v15n25p262

\section{Résumé}

La signature de contrats de travail de courtes périodes semble être devenue la norme depuis plusieurs décennies. Plusieurs raisons sont évoquées pour expliquer cela : la faiblesse de la demande à laquelle font face les entreprises à côté d'une paupérisation croissante, l'exigence de rentabilité des actionnaires, la concurrence exacerbée, la robotisation, l'offre de travail supérieure à la demande de travail, etc. L'objectif de cet article est de comprendre pourquoi certains travailleurs bénéficient d'emplois stables en dépit des raisons évoquées. Pour atteindre cet objectif, l'article s'est appuyé sur les données de l'ENSESI $2016^{9}$ en Côte d'Ivoire. A l'aide d'un modèle probit binaire avec sélection, l'article conclut qu'un niveau d'études de type supérieur, mais également la connaissance d'une autre langue autre que la langue officielle, le maniement des NTIC désormais des critères dans les curricula sont les facteurs explicatifs significatifs de l'obtention des emplois stables après une période de deux ans. Les travailleurs exerçant dans les secteurs de l'industrie et des services, de même que ceux travaillant dans des entreprises avec présence syndicale ont une probabilité plus grande d'obtenir des emplois stables. Forts de ces résultats, l'article recommande des formations de niveau supérieur, la mise en place par les autorités publiques de mesures d'assouplissement pour l'acquisition de matériels informatiques dans les centres de formations et/ou de manière individuelle afin que l'apprentissage informatique soit plus pratique que théorique de même que l'apprentissage des langues étrangères indispensables sur un marché du travail mondialisé.

Mots clés : Emploi stable, Niveau d'études, NTIC, Langue étrangère, Probit binaire avec sélection

${ }^{9}$ Enquête Nationale sur la Situation de 1'Emploi et le Secteur Informel 


\title{
Analysis of Determinants of Stable Jobs in Cote d'Ivore
}

\author{
N'Gratier Antoine, Docteur en Sciences Economiques
}

Enseignant-chercheur, UFR des Sciences Economiques et de Gestion

Université Félix Houphouët Boigny d'Abidjan, Côte d'Ivoire

\begin{abstract}
The signing of short-term employment contracts seems to have become the norm for several decades. There are several reasons to explain this: the weakness of demand that companies are facing alongside with increasing impoverishment, the requirement of shareholder profitability, the cut-throat competition, robotization, the labor supply superiority to the labor demand, etc. This paper focuses on understanding why some workers have stable jobs despite the reasons given. To achieve this goal, data was obtained from 2016 National Survey of the Situation of Employment and the Informal Sector (NSSEIS) in Côte d'Ivoire. With the help of a binary probit model based on selection, the paper concludes that a higher level of education, the knowledge of another language other than the official language, and the knowledge of NICT which is a criteria in curricula are significant explanatory factors for obtaining stable employments after a two-year period. The workers in the industrial and service sectors, as well as workers in union-based firms are more likely to obtain stable jobs. Based on these results, the paper recommends higher level of training and the implementation of easing measures by the public authorities for the acquisition of computer equipment in the training centers and / or individually. This would make the computer learning more practical than theoretical in learning the foreign languages needed in a globalized labor market.
\end{abstract}

Keywords: Stable job, Level of studies, NICT, Foreign language, Binary probit with selection

\section{Introduction}

L'évolution des contrats de travail est liée d'une part à l'évolution macroéconomique dans les Etats et d'autre part, à l'évolution des caractéristiques des unités économiques (offreurs et demandeurs de travail).

Jusqu'aux trente glorieuses, les économies du monde baignaient dans une croissance économique soutenue et stable, avec des taux de chômage 
relativement bas et l'existence d'emplois stables ${ }^{10}$. Même dans les pays en voie de développement, on assistait à des niveaux de croissance jamais rencontrés qui auraient dû permettre à ces derniers de quitter définitivement le cercle vicieux de la pauvreté dans lequel ils se trouvaient. Or, après les différents chocs pétroliers, l'instauration des programmes d'ajustements structurels et tout leur corollaire, la quasi-totalité des pays a dû faire face à des ralentissements de leur croissance économique dans le meilleur des cas, quand d'autres se sont vus imposer des mesures d'austérité et des thérapies de chocs pas toujours favorables à leur volet social (Muet, 1991; Nassar, 1993 ; Antonin, 2013).

Ainsi donc, depuis les années 1970, on peut recenser trois faits... qui ont bouleversé les conditions économiques : une «nouvelle » mondialisation qui a accru la concurrence ...; le premier choc pétrolier; le développement de la part des services dans la production (Duhautois et Gonzales, 2013).

La dualité du marché du travail s'est accrue au fil des décennies par un accroissement de la précarité de l'emploi, le recours aux contrats de travail de courtes durées assortis de peu ou pas de perspectives de carrière, et où les conditions de travail sont mauvaises. Ce dualisme accentué du marché du travail venant confirmer l'hétérogénéité de l'offre de travail infirmant l'hypothèse néoclassique d'homogénéité des offreurs de travail.

Les différentes crises récentes: crise des subprimes, les scandales financiers dans les secteurs financier et bancaire qui impactent les autres secteurs d'activités, la financiarisation de l'économie, la mondialisation des échanges qui s'accompagnent de concurrence exacerbée emmènent les entreprises à réduire considérablement leurs coûts de production notamment par un rapiècement de leurs mains d'œuvre quand celles-ci ne ferment pas purement et simplement (Boyer, 2009).

Dans un contexte économique difficile avec un taux de chômage élevé, chercher, trouver et conserver un emploi stable devient une gageure. L'emploi est parfois désiré pour lui-même dans la mesure où il permet de répondre aux besoins primaires et de civilisations. De la stabilité de l'emploi découle une stabilité financière mais surtout sociale. Face aux incertitudes économiques et à la difficulté des anticipations rationnelles, les offreurs de travail qui bénéficient de contrats de travail stables se trouvent dans une position où les incertitudes sont à minima et les anticipations plus ou moins réalisables. Certes, cette assertion peut être contestée quand on sait que certaines formes de contrats de travail instables sont voulues (free-lance, temps partiel voulu, etc.). Toutefois, l'on retient qu'en règle générale celle-là se confirme.

\footnotetext{
${ }^{10}$ Dans cet article, les emplois stables ou permanents désignent les contrats de travail stables ou de longues périodes à la différence des emplois instables qui désignent des contrats de travail instables ou de courtes périodes.
} 
Sur le marché du travail des pays africains, le secteur public n'arrivant plus à faire face à l'absorption de la main d'œuvre, les entreprises du secteur privé ont dû s'adapter pour contenir le trop plein d'offreurs de travail sur le marché. On a assisté dès lors, à une croissance des contrats instables et nonpermanents $^{11}$ : contrats à durée déterminée, contrats à durée déterminée à terme imprécis, contrats d'intérim, contrats temporaires ou occasionnels, etc.

Les données de 2016 en ce concerne la Côte d'Ivoire (ENSESI, 2016) montrent par exemple que plus de $65 \%^{12}$ des travailleurs ont des emplois instables. Les hommes avec $67.44 \%$ de même que les travailleurs ayant un niveau d'études inférieures à l'université avec $90.97 \%$ concentrent la majorité des cas.

En réalité le problème ne se situe pas dans la signature d'un contrat non-permanent tel qu'un Contrat à Durée Déterminée $\left(\mathrm{CDD}^{13}\right)$, mais dans la perpétuation de ce type de contrat au-delà d'une certaine période d'activité en entreprise que cet article a bornée au maximum à deux ans comme c'est le cas des CDD en Côte d'Ivoire. En effet, au sujet de ces types de contrats de travail, le code du travail ivoirien prévoit en son article 15.4 que : «Les contrats à terme précis ne peuvent être conclus pour une durée supérieure à deux ans. Les contrats à terme précis peuvent être renouvelés sans limitation. Toutefois, ces renouvellements ne peuvent avoir pour effet d'entraîner un dépassement de la durée maximale de deux ans.». Cette borne de deux ans maximum retenue s'inscrit dans la même logique et le même esprit tels qu'énoncés par le code du travail ivoirien qui est de ne pas voir des contrats instables et irréguliers s'éterniser.

D’importantes études et rapports sur l'évolution des contrats de travail ont été produits en Côte d'Ivoire. Quelques travaux universitaires ${ }^{14}$ ont décrit l'évolution des formes de contrats de travail et mis en évidence les raisons qui auraient pu entrainer ce changement de paradigme.

La présente recherche qui s'appuie sur les résultats passés tente de comprendre aujourd'hui, ce qui caractériserait les travailleurs bénéficiant d'emplois stables après la période sus-indiquée, supposition étant faite que les emplois stables assureraient une vie décente et procureraient par la même occasion une meilleure productivité aux entreprises. Il faut voir dans cette relation de travail stable, un gain à la fois pour l'employé et pour l'employeur. Mais comment en arriver à la signature d'un contrat de travail stable avant la

\footnotetext{
${ }^{11}$ Une liste non exhaustive des typologies de contrats flexibles est présentée en annexe.

${ }^{12} \mathrm{Cf}$ annexe.

${ }^{13}$ Dans cette étude, les CDD, les intérims et tout autre type de contrat autre que les Contrats à Durée Indéterminée (CDI) seront assimilés aux contrats de travail instables. Les CDI quant à eux seront considérés comme des contrats de travail stables.

${ }^{14}$ Le marché du travail du secteur moderne en Côte d'Ivoire : de la rigidité à la flexibilité ; thèse de doctorat de Kouadio Benié Marcel (1995).
} 
période de deux ans (dans le meilleur des cas) ou au terme de cette période? Autrement dit, en considérant deux travailleurs $\mathrm{A}$ et $\mathrm{B}$ embauchés à la même date en CDD par exemple, qu'est ce qui expliquerait qu'au terme de ce CDD, le travailleur A obtienne un CDI et que le travailleur B demeure toujours en $\mathrm{CDD}^{15}$ ?

De là, découle la question de recherche suivante : pourquoi certains travailleurs bénéficieraient-ils d'emplois stables après avoir travaillé plus de deux ans pour leur employeur, et d'autres pas ? Autrement dit, quel profil ont les travailleurs qui bénéficient des emplois stables après une période de deux ans en entreprise.

L'objectif de l'article consiste à analyser les déterminants des emplois stables chez les travailleurs de Côte d'Ivoire.

\section{II- Revue de littérature}

Dans cette revue de littérature, cet article montrera d'une part les raisons qui amèneraient les entreprises à recourir aux contrats instables, et d'autre part pourquoi malgré cette tendance des entreprises à l'offre de contrats instables, il y a certains travailleurs qui arrivent à obtenir des emplois stables.

Le cadre théorique mobilisera les théories des coûts de transactions, de l'agence et des contrats implicites, puis seront présentés les raisons qui conduisent les entreprises à la signature de contrats courts et enfin seront exposés les travaux empiriques qui justifient le fait que certains salariés bénéficient de contrats de travail stables, malgré la tendance à l'offre d'emplois instables de la part des employeurs.

\section{II-1 La problématique des incertitudes sur le marché du travail : les théories de l'agence, des contrats implicites et des coûts de transactions.}

La théorie de l'agence tente de répondre à la question de l'aléa moral dans la mesure où dans une relation entre deux parties, l'une est susceptible d'avoir un comportement qui nuirait à l'autre. Cette théorie analyse les situations dans lesquelles une partie prenante appelée agent (ou mandataire) affecte par son comportement, les intérêts de l'autre partie prenante appelée principal (ou mandant). Dans cette relation où règne des incertitudes, le problème pour le principal serait de mettre en place un système incitatif de sorte que l'agent développe des actions dans l'intérêt du premier (Moureau et Rivaud-Danset, 2004). Dans la relation de travail, face à l'incertitude qui pèse sur la probabilité de trouver un emploi et l'incertitude quant à l'effort maximal qu'est susceptible de déployer l'agent (le travailleur), le principal

${ }^{15}$ Bien entendu cette situation doit être dénoncée puisque le code du travail ivoirien fixe la norme en la matière. 
(l'employeur), pour réduire l'aléa moral lié à l'effort du premier peut proposer un emploi stable qui l'empêcherait de minimiser ses efforts et constituerait un manque à gagner au cas où il le perdrait. Cette adaptation de la théorie de l'agence supposerait en effet que le travailleur est averse à l'instabilité de l'emploi.

La théorie des contrats implicites qui s'inscrit dans le même raisonnement de la problématique des incertitudes postule que face à l'incertitude sur le marché des biens et services qui implique un risque de chômage pour le travailleur et un risque de débouchés pour l'employeur, le principal et l'agent préfèrent arriver à la signature d'un contrat de travail où la rémunération serait certes faible, mais stable ce qui garantirait une forte sécurité de l'emploi quel qu'en soit la conjoncture. En faisant l'hypothèse que l'agent est averse au risque de chômage, celui-ci bénéficierait d'un salaire $\omega_{0}$ $<\omega^{*}$ de sorte qu'en situation de mauvaise conjoncture le même salaire $\omega_{0}$ lui soit versé. En principe, en l'absence de contrat implicite entre le principal et l'agent, l'employeur qui joue un rôle d'assureur proposerait un salaire élevé en cas de risque de chômage fort. Or tel n'est pas le cas en présence de contrats implicites. Ainsi donc, en situation de conjoncture favorable, le principal supposé indifférent face au risque bénéficie d'un différentiel de salaire qui aurait dû être versé à l'agent si le libre jeu du marché guidait la relation de travail (Drèze, 2000 ; Lesueur et Sabatier, 2008).

O. Williamson (1975) s'appuyant sur les travaux de R. Coase et H. Simon quant aux relations qui pourraient exister au sein de la firme et du marché, apporte plus de précisions auxdites relations. Dans celles-ci entre agents et principaux ce n'est plus tant l'échange qui prévaut mais la transaction qui va au-delà de celui-ci en introduisant la notion de temps. La signature de contrats de travail (supposés stables) dans le temps permet d'éviter des comportements opportunistes et donc de réduire des coûts de transactions de part et d'autre des parties en contrat. L'absence de contrat de travail pourrait d'une part conduire les travailleurs à déployer moins d'efforts supposés observables à partir des objectifs, et d'autre part inciter les employeurs à faire varier à la baisse les niveaux de rémunération ou à mettre fin à la relation de travail unilatéralement.

Ces trois théories qui essaient d'apporter des réponses à la problématique des incertitudes, expliquent les raisons pour lesquels agent et principal préfèrent arriver à la signature de contrats de travail qui minimiseraient le moral hazard. Toutefois, la théorie des contrats implicites éclaire au mieux l'arbitrage entre niveau de salaire et degré de stabilité dans l'emploi. Si ces théories ont le méritent de faire comprendre les nœuds de contrats, il faut remarquer que la configuration actuelle des économies et les travaux empiriques sur l'instabilité des emplois, la flexisécurité de ceux-ci montrent une tout autre approche de la gestion des incertitudes. Mieux, il 
semble qu'en présence d'incertitudes multiples tant sur le marché des biens et services, du travail, de la monnaie, etc. les employeurs soient amenés à proposer de plus en plus de contrats courts, flexibles, etc.

\section{II-2 Pourquoi les employeurs ont-ils de plus en plus recours aux contrats de travail courts?}

Le recours par les entreprises aux contrats de courtes périodes devrait servir de test avant l'embauche à durée indéterminée. Ce recours s'explique aussi par le fait de vouloir faire face à l'incertitude et aux fluctuations de la demande (Bunel, 2007). En principe le contrat court, CDD par exemple, constituerait un tremplin pour les travailleurs en vue d'accéder aux contrats à durée indéterminée (CDI). Or, aujourd'hui, l'on assiste à une utilisation abusive des CDD. Certaines entreprises en sont arrivées à faire signer à leurs salariés en fin de CDD des contrats de travail à durée déterminée à terme imprécis, contournant ainsi la loi tout en ne se la mettant pas à dos.

Plusieurs raisons expliquent le recours aux contrats de travail courts de la part des employeurs. Nous pouvons citer entre autres la morosité du contexte économique mondial, l'exigence de la clientèle, l'automatisation des tâches, le chômage de masse et le comportement des salariés.

Le contexte économique mondial depuis les différents chocs pétroliers, la mise en place des programmes d'ajustement structurels et le ralentissement de la croissance ont conduit les entreprises des pays du Sud à réduire leurs coûts de production, notamment leurs mains d'œuvre (Duhautois et Gonzales, 2013, op. cit.). La baisse de la demande de la part des consommateurs couplée à de la concurrence exacerbée n'ont fait qu'accentuer cette réduction drastique des coûts de production.

Les entreprises sont désormais à la recherche d'adaptabilité face à une économie de marché de plus en plus incertaine. De plus, la financiarisation de l'économie qui conduit à une forte mobilité des capitaux et la pression des actionnaires ne font que contribuer à faire peser sur les entreprises la recherche de programmes de minimisation de leurs coûts de production. On assiste dès lors à des recours aux emplois partiels, temporaires, intérimaires dans le meilleur des cas, sinon à des dégraissages et licenciements techniques purs et simples.

Par ailleurs, les habitudes de consommation ont fortement évoluées (?) au point de conduire à des exigences de consommation de la part de la clientèle. Par exemple, on assiste aujourd'hui à des ouvertures de points services (supermarchés, agences bancaires et de télécommunication, etc.) à des heures décalées ou du dimanche et des jours fériés. L'introduction de ces formes de flexibilité interne de travail par les entreprises conduisent celles-ci à la formation d'équipes de jour et de nuit avec parfois des contrats de travail instables tenant compte desdites formes de flexibilité. 
Aujourd'hui, l'automatisation et la robotisation des tâches laissent peu de marge de négociation aux travailleurs. Face à la technologie, se voir offrir un emploi même instable pourrait être une véritable aubaine ! Les entreprises investissent de plus en plus dans la technologie de pointe en vue de réduire les coûts de la main d'œuvre. Le secteur des services notamment le domaine de la banque en est une parfaite illustration. L'introduction de Guichets Automatiques de Billets (GAB) a considérablement modifiée la structure de fonctionnement des points de vente. Les tâches habituellement dévolues aux guichetiers-payeurs (retraits, dépôts, traitement de virements, demande de chéquiers, etc.) sont de plus en plus confiées aux GAB qui, une fois installés et bien révisés techniquement offrent un service très apprécié. Si la technologie et la robotisation avaient été introduites en vue d'appuyer la main d'œuvre, il faut désormais souligner que celles-ci se posent en véritables concurrentes et tendent à ravir la place qui était auparavant celle des travailleurs.

A cette automatisation et robotisation des tâches, il faut ajouter le niveau élevé de chômage qui n'est pas de nature à rendre la tâche aisée aux travailleurs. En effet, celui-ci a profondément modifié l'équilibre sur le marché du travail voire le rapport de négociation. Les travailleurs sont dans une posture d'acceptation des offres d'emplois qui se présentent à eux. Face à une main d'œuvre qui grossit d'année en année et à une raréfaction de l'emploi, les travailleurs peuvent se voir obliger d'accepter des contrats instables.

Enfin, le comportement des salariés peut expliquer le recours par les employeurs aux emplois instables. Le développement du freelance, de l'autonomie dans le travail poussent certains salariés à opter pour des emplois de courtes durées, certes instables, mais moins exigeante.

\section{II-3 Caractéristiques des travailleurs, environnement de travail et contrats de travail}

Si les raisons évoquées dans les lignes qui précèdent expliquent le recours par les employeurs aux contrats instables, il est à noter que tous les travailleurs ne subissent pas le choix de tels types de contrats. Les caractéristiques intrinsèques de ceux-ci, les aptitudes acquises et l'environnement dans lequel chaque travailleur évolue expliquent en partie le fait d'avoir un contrat de travail court ou un contrat de travail long.

Lopez (2004) dans son article sur «Les modes de stabilisation en emploi en début d'activité » montre que le niveau et le type de diplôme peuvent favoriser l'acquisition d'emplois stables. En effet, dans son étude, il arrive à la conclusion que les titulaires des diplômes les plus prestigieux accèdent aux contrats à durée indéterminée contrairement aux diplômés de 
niveau inférieur. De plus, les formations préparant aux métiers industriels offrent plus de chances d'avoir un emploi stable.

Moncel (2012) arrive à une conclusion quasi-similaire lorsqu'elle montre que la détention d'un diplôme de type du supérieur garantirait à long terme un emploi de qualité supérieur par rapport au début de la carrière.

Bunel (2007) montre à partir de certains faits stylisés qu'en France le niveau de diplôme accroît très fortement la probabilité d'obtenir un CDI après une période de Contrat à Durée Limitée (CDL). L'écart entre les individus qui ont un diplôme niveau BAC+2 et plus et ceux qui ne disposent que d'un niveau BEPC est de plus de 10 points.

L'âge du travailleur a également une influence sur sa probabilité de bénéficier d'un emploi stable. Baranski (2014) sur données européennes, en analysant les déterminants de l'emploi temporaire en Pologne conclut que les emplois instables sont la plupart du temps occupés par les jeunes travailleurs de moins de 30 ans. Toutefois, autour de 30 ans, la probabilité d'obtenir des emplois instables diminue mais moins rapidement.

Ben Halima (2005) arrive aussi à une conclusion identique dans un article sur les déterminants de la durée des contrats de travail. Il met en évidence la relation décroissante entre l'âge et l'obtention d'un contrat court.

Bunel (2007) trouve un résultat contraire à Baranski. En effet, il souligne dans une tout autre sphère que les CDI après des CDL sont la plupart du temps occupés par les travailleurs de 25 ans et moins.

Tous ces travaux mettent en avant le niveau d'études et l'âge comme déterminants de l'acquisition de contrats de travail stables. Comme cela sera vu dans les lignes qui suivent, cet article va au-delà de ces déterminants en montrant que les connaissances liées aux NTIC et à la pratique des langues autre que la langue officielle participent à augmenter la probabilité d'obtenir des emplois stables.

\section{III- Méthodologie}

\section{III-1 Un modèle théorique des contrats de travail stables}

Le modèle théorique ci-après tiré de Cahuc et Zylberberg (1996) pp. 258-262, Lazear et Rosen (1981), Malcomsom (1984) tels que cités par Stankiewicz (1999) tente d'adapter le modèle de concurrence entre salariés pour l'acquisition d'un supplément de salaire à celui de l'acquisition d'un contrat de travail stable. Le modèle montre que la possession de certaines compétences du travailleur conduit ce dernier à bénéficier d'un contrat de travail permanent et stable se matérialisant par un salaire plus élevé.

La production du salarié dépend de la somme de performances $\sigma(\kappa)$ que l'on suppose être dépendante positivement de ses qualifications $(\kappa)$ (niveau d'études, connaissances des NTIC, connaissances de langues étrangères, autoapprentissage) toutes choses étant égales par ailleurs, et d'une 
variable aléatoire (« la chance »), de moyenne égale à zéro, dont l'employeur connaît la loi de probabilité. Soit $q=\sigma+\theta$.

Le système incitatif est le suivant : le salarié recevra un contrat à durée indéterminé au terme de son contrat à durée déterminée et cela se matérialisera par un salaire $(\omega+b)$ où $b$ représente le bonus qui n'est rien d'autre qu'un supplément de rémunération sous forme de promotion lié à l'obtention d'un Contrat à Durée Indéterminée si sa production est au moins égale à $\bar{q}$ (donc si $q \geq \bar{q}$, soit si $\theta \geq \bar{q}-\sigma$ ) et il recevra $\omega$ si sa production est inférieur à $\bar{q}$.

Le problème pour l'employeur est de fixer, de façon optimale, $\omega, b$ et $\bar{q}$ de façon à maximiser son profit, compte tenu de la maximisation par le salarié de sa propre utilité.

\section{1- Le choix optimal de $\sigma^{*}$ du salarié}

Le salarié percevra $b$ si $\theta \geq \bar{q}-\sigma$. La probabilité de recevoir le bonus est égale à $[1-F(\bar{q}-\sigma)]$ où $F$ désigne la fonction de répartition de $\theta$. $F(\bar{q}-\sigma)$ est ainsi la probabilité que $\theta$ soit inférieur à $(\bar{q}-\sigma)$. L'espérance d'utilité $U$ du salarié, supposé neutre au risque, est donc égale à :

$U=\omega+b[1-F(\bar{q}-\sigma)]-c(\sigma)$ où $c(\sigma)$ désigne la désutilité de la somme des performances avec $c^{\prime}>0$ et $c^{\prime \prime}>0$.

Le niveau des performances $\sigma^{*}$ qui maximise $U$ est tel que $\delta U / \delta \sigma=0$ , soit : $b f\left(\bar{q}-\sigma^{*}\right)=c^{\prime}\left(\sigma^{*}\right)$ où $f$ est la densité de probabilité de $\theta$, en l'occurrence la probabilité que $\theta$ soit égal à $\left(\bar{q}-\sigma^{*}\right)$.

\section{2- La maximisation du profit de l'entreprise}

L'espérance du profit par salarié, $\pi$, est égale à :

$$
\pi=\sigma-\omega-b[1-F(\bar{q}-\sigma)] .
$$

$\mathrm{Si}$ on suppose que l'employeur n'accorde pas plus au salarié que ce que ce dernier pourrait obtenir ailleurs, on a :

$$
U=U(a) \text {, soit: } \omega+b[1-F(\bar{q}-\sigma)]=c(\sigma)+U(a)
$$

L'espérance du profit, $\pi$, est donc : $\pi=\sigma-[\omega+b[1-F(\bar{q}-\sigma)]$

$$
\begin{gathered}
=\sigma-c(\sigma)-U(a) \\
=\sigma-c(\sigma)-[\omega+b(1-F(\bar{q}-\sigma))-c(\sigma)] \\
=\sigma-\omega-b[1-F(\bar{q}-\sigma)]
\end{gathered}
$$

On retrouve l'expression de départ de manière triviale.

L'espérance de $\pi$ est maximale pour $\delta \pi / \delta \sigma=0$, soit $c\left(\sigma^{*}\right)=1$.

\section{3- Les valeurs optimales de $\omega, b$ et $\bar{q}$}

Elles devront satisfaire aux conditions :

$$
b f\left(\bar{q}-\sigma^{*}\right)=1 \text { et } \omega+b\left[1-F\left(\bar{q}-\sigma^{*}\right)\right]=c\left(\sigma^{*}\right)+U(a)
$$


Le fait qu'une proportion de salariés recevront $(\omega+b)$, les autres n'obtenant que $\omega$, peut s'interpréter comme l'octroi d'un bonus salarial lié (comme signifié plus haut) au bénéfice d'un contrat de long terme ou promotion hiérarchique d'un emploi payé $\omega$ à un emploi rémunéré $(\omega+b)$. Une augmentation de la variance de $\theta$ induirait une augmentation de $b$, ce qui impliquerait une réduction de la proportion $[1-F(\bar{q}-\sigma)]$ des bénéficiaires. Par ailleurs, une aversion pour le risque aurait pour effet de baisser $b$.

\section{III-2 Modèle économétrique : le probit binaire avec sélection}

Le modèle que cet article utilise pour les estimations est un modèle probit binaire avec sélection. Il permettra de voir l'influence de certaines caractéristiques du travailleur et de son environnement de travail sur sa probabilité à obtenir un emploi stable. Il s'inspire des travaux de Heckman (1979) et peut être formalisé comme suit pour chaque individu de l'échantillon :

- Expérience professionnelle dans l'entreprise supérieure à 2 ans (équation de sélection) $: z_{i}^{*}=w_{i} \alpha+\mu_{i}$ où $z_{i}^{*}$ est observé uniquement si l'individu $i$ a une expérience professionnelle supérieure à 2 ans.

De manière plus détaillée, l'équation de sélection se présente comme suit :

$\operatorname{EXPPROENTR}_{i}=\alpha_{0}+\alpha_{1} S E X_{i}+\alpha_{2} A G E_{i}+\alpha_{3} E D U_{i}+$ $\alpha_{3}$ TYPFORM $_{i}+\alpha_{4}$ TYPENTR $_{i}+\alpha_{5}$ MILRESID $_{i}+\mu_{i}$

Où pour l'individu $i$, on a:

EXPROENTR représente l'expérience professionnelle passée en entreprise et dépend des variables explicatives suivantes : $S E X$ représente le sexe, $A G E$ représente l'âge, $E D U$ le niveau d'études, $T Y P F O R M$ le type de formation, TYPENTR représente le type d'entreprise et MILRESID le milieu de résidence.

- Occupation d'un emploi stable (équation substantielle) : $y_{i}^{*}=x_{i} \beta+\varepsilon_{i}$ observable uniquement si $z_{i}^{*}>0$.

L'équation substantielle se présente comme suit :

EMPLSTAB $B_{i}=\beta_{0}+\beta_{1}$ SEX $_{i}+\beta_{2}$ SITMAT $_{i}+\beta_{3} E D U_{i}+$ $\beta_{3}$ CONNAUTRLANG $_{i}+\beta_{4}$ CONNINFO $_{i}+\beta_{5}$ EXPPRO $_{i}+$ $\beta_{6}$ MODOBTEMPL $_{i}+\beta_{7}$ TAILENTR $_{i}+\beta_{8}$ BRANCACT $_{i}+$ $\beta_{9} S Y N D I C_{i}+\varepsilon_{i}$

Où pour l'individu $i$, on a :

EMPLSTAB pour emploi stable dépend des variables explicatives suivantes : SEX pour sexe, SITMAT pour situation matrimoniale, EDU pour niveau d'éducation, CONNAUTRLANG pour connaissance d'une autre langue, CONNINFO pour connaissance de l'informatique, EXPPRO pour expérience professionnelle dans l'emploi, MODOBTEMPL pour mode d'obtention de l'emploi, TAILENTR pour 
taille de l'entreprise, BRANCACT pour branche d'activité, SYNDIC pour présence syndicale en entreprise.

Avec $\mu_{i}$ suivant une loi normale $N(0 ; 1)$ et $\varepsilon_{i}$ suivant une loi normale $N\left(0 ; \sigma_{\varepsilon}\right)$.

La régression est effectuée en deux étapes avec l'estimateur du maximum de vraisemblance sous Stata et avec la commande « heckprob». Avec cette méthode et cette fonction, une correction robuste de l'hétéroscédasticité des erreurs types est effectuée. Le ratio de Mills proposé par Heckman est inclus dans l'équation substantielle en vue de la correction du biais de sélection.

Le test de Chi2 permet de se rendre compte que l'équation de sélection et l'équation substantielle sont indépendantes ou non. Les résultats de l'estimation permettent de rejeter l'hypothèse nulle d'indépendance des équations si la pvalue $<0,05$ (seuil critique).

\section{III-3 La source de données et les variables du modèle}

Les données utilisées dans cet article proviennent de l'Enquête Nationale sur la Situation de l'Emploi et le Secteur Informel (ENSESI, 2016). Cette enquête a été diligentée par l'Etat de Côte d'Ivoire à travers le Ministère de l'Emploi et de la protection sociale, de l'Institut National de la Statistique et l'Agence Emploi Jeunes. Comme indiqué par l'Agence Emploi Jeune:«L'ENSESI 2016 est une opération statistique à deux phases. La première phase est une enquête permettant de collecter des données sur les caractéristiques socio démographiques et sur l'emploi. Cette première phase sert de filtre à la seconde qui est une enquête du type «entreprise » réalisée auprès des Unités de Production Informelles (UPI) non agricoles identifiées au cours de la première phase. Cette opération est en fait une variante du système d'enquêtes 1-2-3 pour laquelle la phase 3 n'est pas réalisée. »

L'ENSESI renferme l'ensemble des variables qui servent de calculs et d'estimation pour le modèle. Le nombre total d'observations 2279 auquel cette recherche parvient se justifie par le fait qu'ont été exclus de l'échantillon l'ensemble des individus n'ayant pas 15 ans pour répondre à une exigence mondiale de lutte contre le travail des enfants. De plus, ont été exclus de l'échantillon tous les individus n'ayant pas répondu à la question de savoir quel était leur type de contrat de travail.

Les variables du modèle sont codés comme suit :

- La variable expliquée, pour l'équation de sélection, il s'agit d'avoir (1) ou non (0) une ancienneté dans l'entreprise supérieure à deux ans. Pour l'équation substantielle, il s'agit d'avoir un emploi stable qui vaut 1 si oui et 0 si non.

- Les variables explicatives : 
- Le sexe : variable codée 1 pour homme et 2 pour femme. L'on pourrait s'attendre à ce que les hommes soient discriminés positivement par rapport aux femmes, tant dans l'équation de sélection que dans l'équation substantielle.

- La situation matrimoniale : codée 1 pour marié, 2 pour divorcé/séparé, 3 pour veuf/veuve et 4 pour jamais marié/Célibataire. Avec cette variable présente uniquement dans l'équation substantielle, l'on devrait s'attendre à ce que les personnes mariées soient ceux qui occupent des emplois stables en raison de leur statut matrimonial qui pourrait s'apparenter à une certaine stabilité.

- Le niveau d'éducation : variable codée 1 pour aucun niveau, 2 pour niveau primaire, 3 pour niveau secondaire et 4 pour niveau supérieur. Le niveau d'éducation présent aussi bien dans l'équation de sélection que dans l'équation substantielle devrait avoir une significativité importante. En effet, on s'attend à ce qu'un niveau d'études supérieur impacte positivement la probabilité d'obtenir un emploi stable.

- La connaissance des langues autre que la langue officielle (le français): codée 0 pour aucune connaissance, 1 pour connaissance par la lecture uniquement et 2 pour connaissance par la lecture et l'écriture. Uniquement intégrée dans l'équation substantielle, la connaissance des langues autre que la langue officielle pourrait augmenter la probabilité d'obtenir un emploi stable.

- La connaissance de l'informatique: codée 0 pour aucune connaissance, 1 pour bonne connaissance et 2 pour connaissance moyenne. Uniquement intégrée dans l'équation substantielle, cette variable pourrait influencer positivement la probabilité d'obtenir un emploi stable.

- L'expérience dans la profession ${ }^{16}$ : codée 1 pour $(0-2)$ ans, 2 pour (3$10)$ ans et 3 pour (11 ans et + ). Cette variable intégrée uniquement dans l'équation substantielle pourrait avoir un effet positif sur la probabilité d'avoir un emploi stable. En effet, pour éviter un coût lié à la " perte » de l'employé ayant accumulé de l'expérience professionnelle, l'entreprise pourrait être amenée à lui concéder un emploi stable. La transférabilité de l'expérience vers une autre entreprise serait un manque à gagner pour la première.

- La taille de l'entreprise : codée 0 pour ne sait pas, 1 pour 1 seule personne, 2 pour (2-5) personnes, 3 pour (6-9) personnes, 4 pour (10199) personnes et 5 pour plus de 199 personnes. Uniquement dans

\footnotetext{
${ }^{16}$ A ne pas confondre avec l'expérience professionnelle passée dans l'entreprise au moment de l'enquête. L'expérience dans la profession indique l'expérience acquise dans l'emploi (qui peut être la somme des expériences dans divers entreprises mais au même poste d'activité).
} 
l'équation substantielle, l'on pourrait s'attendre à ce qu'un travailleur exerçant dans une entreprise de taille importante ait une probabilité plus grande d'obtenir un emploi stable. Cependant, cette corrélation devrait être mitigée.

- La branche d'activité : codée 1 pour agriculture, 2 pour industrie, 3 pour commerce et 4 pour service. Avec cette variable présente dans l'équation substantielle, la prédiction s'avère délicate.

- L'existence de syndicat en entreprise : 1 pour oui et 2 pour non. Cette variable est uniquement présente dans l'équation substantielle. Le rôle du syndicat dans l'obtention d'un emploi stable pourrait s'avérer importante.

- L'âge : variable codée 1 pour (15-24) ans, 2 pour (25-35) ans, 3 pour (36-59) ans et 4 pour (60 ans et + ). Difficile de prédire le sens de la probabilité avec cette variable présente uniquement dans l'équation de sélection.

- Le type de formation : variable codée 0 pour autre type de formation, 1 pour formation général, 2 pour formation technique et 3 pour formation professionnelle. Uniquement présente dans l'équation de sélection, il serait hasardeux de prédire qu'un travailleur ayant subi une formation de tel ou tel type aurait une probabilité plus grande d'avoir plus de deux ans d'expérience dans l'entreprise.

- Le mode d'obtention de l'emploi : variable codée 1 pour concours, 2 pour réseau Parents-Amis-Connaissances, 3 pour petites annonces, agences privées de placement, 4 pour structures publiques (AGEPE, $\mathrm{FNS}^{17}$, etc.). Il est difficile de faire une prédiction avec cette variable uniquement présente dans l'équation de sélection.

- Le type d'entreprise : variable codée 1 pour administration ou entreprise publique ou parapublique, 2 pour entreprise privée non agricole, 3 pour entreprise privée agricole et 4 pour autres entreprises. La variable type d'entreprise est uniquement présente dans l'équation de sélection. L'on pourrait s'attendre à ce que les travailleurs exerçant dans une administration publique ou parapublique aient une probabilité plus grande d'avoir un emploi de plus de deux ans dans leur entreprise. Mais le statut de certains employés du publique notamment les agents de l'Etat n'autorise pas à faire une telle prédiction.

- Le milieu de résidence : variable codé 1 pour Abidjan, 2 pour autres urbains et 3 pour rural. Avec cette variable dans l'équation de sélection, l'on pourrait s'attendre à ce que les travailleurs exerçant

${ }^{17}$ Fonds National de Solidarité. 
dans les milieux urbains aient une probabilité plus grande d'obtenir un emploi d'une durée de plus de deux ans.

\section{IV- Interprétation des résultats}

Les interprétations concerneront les statistiques descriptives d'une part et d'autre part, les estimations économétriques.

\section{IV-1 Statistiques descriptives}

Les statistiques descriptives sont celles de l'ensemble de la population et celles de la sous-population des personnes ayant un emploi stable.

Au sujet de l'ensemble de la population constituée de 2279 individus, l'on note globalement qu'il y a plus de personnes ayant une ancienneté dans leur entreprise qui date de plus de deux (02) ans.

Dans ce lot et en termes de pourcentage, les travailleurs ayant une ancienneté de plus de deux ans ont les caractéristiques suivantes : travailleurs hommes, âgés de plus de 36 ans, ayant un niveau d'études supérieures, ayant effectué une formation technique, exerçant dans une entreprise publique ou parapublique et résidents à Abidjan. A partir de ces premières statistiques, l'on peut énoncer quelques explications liées aux différents pourcentages. La nature du marché du travail lié au chômage élevé empêche les primo demandeurs jeunes à y accéder en grand nombre comparativement au plus âgés. Ces derniers (les 36 ans et plus) ont-ils certainement exercé dans quelque(s) entreprise(s) avant celle à laquelle ils appartiennent au moment de l'enquête ? Ce qui leur vaut une chance plus grande d'avoir une durée plus grande que les plus jeunes. Le niveau d'études joue un rôle important dans la durée de présence dans l'entreprise. Les travailleurs ayant un niveau d'études supérieures sont les plus nombreux à bénéficier d'une durée dans l'entreprise de plus de deux ans. Les employeurs préfèrent-ils des travailleurs de ce type en raison du niveau de capital humain acquis qui est certainement lié à un niveau élevé de compétences ou de productivité. Les travailleurs ayant effectué une formation technique bénéficient le plus d'une longue durée en entreprise comparativement aux autres. La formation technique étant certainement plus désirée en raison de son caractère pratique.

Dans le lot de la sous-population des travailleurs occupant un emploi stable, les travailleurs y bénéficiant ont les caractéristiques suivantes : travailleurs femmes, mariés, ayant un niveau d'études supérieures, connaissant une langue autre que le français, connaissant l'informatique, ayant une expérience professionnelle de onze (11) ans et plus, ayant acquis leur emploi par voie de concours ou tests, exerçant dans une grande entreprise, exerçant dans les services ou l'industrie, travaillant dans une entreprise avec présence syndicale. Cette fois, les femmes occupent le plus des emplois stables 
par rapport aux hommes. La féminisation des emplois et la recherche de l'égalité dans le genre peut certainement expliquer cet état de faits. Le travailleur marié pourrait refléter une certaine stabilité dans sa vie personnelle qui se traduirait dans son espace de travail par une certaine organisation, pourraient ainsi conclure les employeurs. Le niveau d'études vient confirmer les thèses jusque-là développées sur l'importance du capital humain. Les mutations qui ont cours dans les milieux professionnels de même que l'internationalisation des connaissances professionnelles emmèneraient les travailleurs à s'adapter. De fait, les connaissances dans les nouvelles technologies de l'information et de la communication couplés parfois à la connaissance d'une autre langue deviennent des atouts pour s'insérer sur le marché du travail et y rester durablement. A cet effet, les statistiques de cette recherche viennent révéler cette assertion. La présence syndicale joue un rôle important dans la durabilité de la relation de travail. Or, l'une des conclusions auxquelles aboutissent les théories économiques sur le marché du travail, c'est que les syndicats certes influencent l'emploi, mais bien plus recherchent l'amélioration du niveau des salaires et de meilleures conditions de travail.

\section{IV-2 Résultats économétriques}

L’interprétation des résultats économétriques se fera aussi bien à partir de l'équation de sélection que de l'équation substantielle.

L'on procède d'abord au test d'indépendance du Khi Deux sur les équations de sélection et substantielle. Pour cela l'on teste l'hypothèse nulle telle que :

$H_{0}$ : L'équation de sélection et l'équation substantielle sont indépendantes

$H_{a}$ : L'équation de sélection et l'équation substantielle ne sont pas indépendantes.

La règle de décision est la suivante : si $x_{o b s}^{2}<x_{l u}^{2}$ on accepte $H_{0}$, c'est-à-dire que les deux équations sont indépendantes. Dans le cas contraire, on rejette $H_{0}$ et on conclut que les équations ne sont pas indépendantes, ce qui implique que les deux décisions ne sont pas prises indépendamment l'une de l'autre; de ce fait la correction du biais de sélection s'en trouve justifiée.

La variable dépendante dans l'équation de sélection possède deux caractères : $p=2$. La variable dépendante dans l'équation substantielle possède elle aussi deux caractères: $q=2$. Le nombre de degrés de liberté est égal à : $(p-1)(q-1)=(2-1)(2-1)=1$ et pour $\alpha=5 \%, x_{o b s}^{2}=30,62$. $x_{l u}^{2}=3,841$. Puisque $x_{o b s}^{2}>x_{l u}^{2}$, on rejette $H_{0}$ et on conclut que les équations ne pas indépendantes.

Conclusion : Les décisions sont prises simultanément et le biais de sélection se trouve justifié. 
Concernant l'équation de sélection, qui est relatif au fait d'avoir une durée dans l'entreprise de plus de deux ans, l'on remarque que les variables explicatives significatives sont : le sexe, l'âge, le type de formation, le type d'entreprise et le milieu de résidence.

La variable sexe est significative au seuil de $1 \%$, l'âge est également significatif au seuil de $1 \%$ pour toutes les modalités, la variable type de formation l'est au seuil de 10\% pour les modalités Général et Professionnel, le type d'entreprise est significatif au seuil de $1 \%$ et $5 \%$ pour les modalités Entreprise privée non agricole, Entreprise agricole et Autres entreprises, respectivement. La variable milieu de résidence est significative au seuil de $10 \%$ pour la modalité Autres urbains.

Les femmes ont une probabilité plus faible que les hommes d'obtenir une durée dans l'entreprise plus grande. Cela semble être lié à leur état de nature biologique: plus absentes en raison de la maternité, de leur statut conjugal, de la « fragilité » de leur état physique ou de santé par rapport aux hommes, etc.

La probabilité d'avoir une durée supérieure à deux ans dans l'entreprise augmenterait avec l'âge du travailleur. Cela serait lié à la compétence acquise au fil des ans par un nouvel entrant. Le coût lié à la séparation d'avec un travailleur ayant exercé pendant une certaine période dans l'entreprise conduirait l'employeur à le (coût) minimiser en faisant en sorte que ce travailleur lui soit fidèle.

$\mathrm{Au}$ sujet de l'équation substantielle, l'article note des résultats forts intéressants. En effet, la probabilité d'obtenir un emploi stable augmenterait avec le niveau d'études ; les travailleurs ayant un niveau d'études supérieures confirment par ce niveau acquis de capital humain que l'investissement en éducation assure de meilleures conditions de travail puisque la probabilité qu'ils obtiennent un contrat de travail stable augmente de 0.2206 point de pourcentage. Les résultats vont au-delà de ce que offre habituellement la littérature, en montrant que la connaissance d'une autre langue et la connaissance de l'informatique sont susceptibles de procurer au travailleur plus de chance d'obtenir un emploi stable. Dans ce cas, la probabilité d'obtention d'un emploi stable augmente respectivement de 0.0842 point de pourcentage et 0.1330 point de pourcentage. Comme dit plus haut, dans les statistiques descriptives, la connaissance des langues et la maitrise de l'outil informatique deviennent incontournables dans un monde professionnel marqué par les échanges et les nouvelles technologies de l'information et de la communication.

Les emplois stables semblent être concentrés dans les grandes entreprises. Les employés travaillant dans ces entreprises, ont 0.1676 point de pourcentage d'obtenir un emploi stable comparativement à ceux exerçant dans une Petite et Moyenne Entreprise (PME) ou dans une Très Petite Entreprise 
(TPE). Ce résultat appelle le questionnement suivant : comment assurer la pérennité des Petites et Moyennes Entreprises en Afrique en général et en Côte d'Ivoire en particulier quand l'on sait que ce sont des unités économiques qui assurent près de $30 \%$ du PIB de ces régions et représentent près de $90 \%$ des entreprises privées du continent?

Les travailleurs exerçant dans les secteurs de l'industrie et des services ont une chance plus grande d'obtenir des emplois stables. Il en est de même de ceux qui travaillent dans des entreprises avec une présence syndicale.

\section{V- Conclusion et recommandations de politiques économiques}

L'objectif de cet article était de comprendre pourquoi certains travailleurs bénéficiaient d'emplois stables et d'autres pas et de mettre en exergue le profil des travailleurs-bénéficiaires. Une revue de littérature comprenant un passage en revue des théories liées à l'incertitude sur le marché du travail, un exposé sur l'évolution des contrats de travail, un questionnement sur le recours des entreprises aux contrats instables et l'exposé de faits empiriques sur ce qui caractériseraient les travailleurs bénéficiant des contrats de travail stables ont permis de cerner quelques contours de la problématique. S'étant aidé d'un modèle probit binaire avec sélection en vue de déceler les facteurs explicatifs qui seraient à l'origine du bénéfice d'un contrat de travail stable, le présent article est arrivé à la conclusion que le niveau d'éducation, la connaissance d'une autre langue autre que la langue officielle (français), la connaissance de l'outil informatique sont autant de facteurs qui permettraient au travailleur en Côte d'Ivoire d'obtenir un emploi stable. Par ailleurs, l'on a remarqué que la présence syndicale joue un rôle important dans l'acquisition d'un contrat de travail stable. Les travailleurs exerçant dans les secteurs de l'industrie et des services ont une chance plus grande d'obtenir des emplois stables.

Conformément à ces résultats, l'article recommande ce qui suit :

- Encourager l'éducation post-secondaire ce qui permettrait aux candidats à l'embauche d'accroître le niveau de leur capital humain en vue de mieux se positionner sur le marché du travail.

- Encourager l'utilisation de l'outil informatique et ce dès l'école primaire. A cet effet, les cours d'informatique dispensés dans les établissements primaires, secondaires ou supérieurs ne devraient pas se limiter à la théorie mais être pratiques. Les initiatives de l'Etat ivoirien en matière de vulgarisation de l'outil informatique par des subventions en vue de l'acquisition d'ordinateurs sont encourageantes. Cependant, cette vulgarisation ne devrait pas se limiter à Abidjan, mais toucher l'ensemble du pays et les coûts d'acquisition des machines revus à la baisse. 
- Encourager la pratique des langues autres que la langue officielle serait un atout supplémentaire pour les candidats à l'embauche en vue de l'obtention de contrats stables. A ce sujet, la mise en place de centres d'apprentissage des langues quasi-universellement parlées (anglais, chinois, arabe, etc.) constituerait un avantage comparatif indéniable surtout pour les primo-demandeurs d'emplois.

- La mise en place par les autorités publiques de mesures incitatives pour les secteurs agricole, le commerce, de même que pour les PME en vue de les encourager à créer des emplois stables et durables.

\section{References :}

1. Antonin, C. (2013). "Après le choc pétrolier d'octobre 1973, l'économie mondiale à l'épreuve du pétrole cher." Revue Internationale et stratégique, 2013/3 n 91, pp 139-149.

2. Auer, P. (2008). "La sécurité du marché du travail : comment conjuguer flexibilité et sécurité pour l'emploi décent ?" Cahiers de l'économie et du marché du travail. Bureau international du travail, Génève.

3. Auer, P. \& Cazes, S. (2002). "Employment stability in an age of flexibility : Evidence from industrialized countries. "Geneva, International Labour Office.

4. Baranski, M. (2014). "Determinants of tempory employment in Poland." Warsaw Forum of Economic Sociology 5 :1(9) Spring 2014, pp 81-110.

5. Behaghel, L. (2003). "Insécurité de l'emploi : le rôle protecteur de l'ancienneté a-t-il baissé en France ?" Economie et statistique, n³66, 2003 ; pp 3-29.

6. Ben-Halima, M.A. (2005). "Les déterminants de la durée des contrats de travail : application micro-économétrique sur l'enquête TDEMLT." UMR-CNRS n ${ }^{\circ} 5824$.

7. Bunel, M. (2007). "Analyser la relation entre CDD et CDI : emboîtement et durée des contrats." Document de travail N82. Centre d'études de l'emploi.

8. BIT (2015). Les formes atypiques d'emploi. Rapport pour discussion à la réunion d'experts sur les formes atypiques d'emploi. Genève, 1619 février 2015.

9. Boyer, M. (2009). "La crise économique et ses conséquences sur l'emploi. Document de travail." Les cahiers de recherche de l'Institut économique de Montréal.

10. Conseil d'orientation pour l'emploi (2014). L'évolution des formes d'emploi. France. 
11. Dallery et al. (2010). "L'incertitude au cœur des marchés concrets. Confrontation entre théorie post-keynésienne et sociologie économique structurale." Innovations 2010/1-n³1 ; pp 131-156.

12. De Nanteuil Miribel, M. (2002). "Les dilemmes de l'entreprise flexible." Document de travail. Université Catholique de Louvain (Belgique) - Laboratoire de sociologie de changements des institutions (France).

13. Drèze, J.H. (2000). "Sur la macroéconomie de l'incertitude et des marchés incomplets." Revue de l'OFCE, nº72; pp 7-37.

14. Duhautois, R. \& Gonzales, L. (2007). "Hétérogénéité des contrats de travail et performances des entreprises en France." hal-00831529.

15. Engellandt, A. \& Riphahn, R.T. (2003). "Temporary contracts and employee effort." Working paper, WWZ-University of Basel, Switzerland.

16. Gasse, D.V. \& Mortelmans, D. (2016). "Contrats de travail flexibles : du concept général à la taxonomie.” Revue belge de sécurité sociale ; pp 229-262.

17. Ghertman, M. (2003). "Oliver Williamson et la théorie des coûts de transaction." Revue française de gestion. 2003/1 n ${ }^{\circ} 142$, pp 42-63.

18. Jaslin et al. (1998). "Du dualisme à la flexibilité du travail." Observations et diagnostics économiques n²3/Avril 1988.

19. Kouadio, B.M. (1995). Le marché du travail du secteur moderne en Côte d'Ivoire : de la rigidité à la flexibilité. Thèse de doctorat, Université Bordeaux 1.

20. Lopez, A. (2004). "Les modes de stabilisation en emploi en début de vie active."Economie et statistique $\mathrm{N}^{\circ} 378-379$, pp 105-128.

21. Lesueur, J.Y. \& Sabatier, M. (2008). Microéconomie de l'emploi : théories et applications. Collection De Boeck ; 256 pages.

22. Moncel, N. (2012). "Quelle qualité d'emploi pour les jeunes diplômés du supérieur?" Formation emploi, Revue française des sciences sociales ; pp 69-87.

23. Moureau, N. \& Rivaud-Danset, D. (2004). L'incertitude dans les théories économiques. Edition la découverte ; 124 pages.

24. Muet, PA. (1991). "Croissance, emploi et chômage dans les années quatre-vingt." Observations et diagnostics économiques n $\mathrm{n}^{\circ} 35$; pp 21 55.

25. Nassar, H.A. (1993). "Quelques conséquences sociales des programmes d'ajustement structurel?" Egypte/Monde arabe, 1213/1993, pp. 145-177.

26. Perez, C. (2014). "La déstabilisation des stables : restructurations financières et travail insoutenable." Travail et emploi, $\mathrm{n}^{\circ} 138$; pp 37 52. 
27. Ramaux, C. (2005). "Les emplois ne sont pas plus instables : explications et incidences sur la régulation de l'emploi." Economies et sociétés, Développement, croissance et progrès-Presses de l'ISMEAParis, 2005, série Socio-Economie du travail, AB (26), pp.1443-1470.

28. Roux, N. (2018). "De l'emploi stable au travail insoutenable, trajectoires d'ouvrières agricoles en groupement d'employeurs." CNAM-CEET, Document de travail $n^{\circ}$ 196, CNAM-CEET.

29. Salladaré, F. \& Hlaimi, S. (2014). "Analysis of the determinants of Temporary employment in 19 European countries." 2014. Hal$00174817 \mathrm{v} 3$.

30. Stankiewicz, F. (1999). Economie des ressources humaines. Edition la Découverte. Répères; 122 pages.

31. Stone, K.V. (2016). "The decline in standard employment contract : evidence from ten advanced industrial countries." Research gate/255724682.

32. Tremblay et al. (1999). "Déterminants du recours au travail atypique : Une étude des travailleurs à statut précaire dans les organisations québécoises." Série scientifique, 99s-39. 


\section{ANNEXE}

Tableau 1 : Différentes formes de flexibilité en interaction (De Nanteuil, 2002).

\begin{tabular}{|c|c|c|}
\hline Flexibilité & Quantitative & Qualitative \\
\hline Externe & \begin{tabular}{cl}
\multicolumn{2}{c}{ Statuts d'emploi } \\
$\checkmark$ & Contrats à durée déterminée \\
$\checkmark$ & Contrats de travail temporaire \\
$\checkmark$ & Stages \\
$\checkmark$ & « autres emplois » \\
& $-\quad$ Emplois subventionnés \\
& $-\quad$ Travail saisonnier \\
& $-\quad$ Travail à la demande \\
$\checkmark$ & Licenciements / Chômage technique \\
& Flexibilité numérique ou contractuelle \\
\end{tabular} & \begin{tabular}{ll} 
& \multicolumn{1}{c}{ Systèmes de production } \\
$\checkmark$ & Sous-traitance \\
$\checkmark$ & Externalisation \\
$\checkmark$ & Travail indépendant \\
& \\
& Flexibilité productive ou géographique
\end{tabular} \\
\hline Interne & \begin{tabular}{cl}
\multicolumn{3}{c}{ Temps de travail et rémunération } \\
$\checkmark$ & Réduction / aménagement de la durée du travail \\
$\checkmark$ & Temps partiel \\
$\checkmark$ & Heures supplémentaires / heures \\
$\checkmark$ & complémentaires \\
$\checkmark$ & Travail posté / travail de nuit / travail de week- \\
$\checkmark$ & end \\
$\checkmark$ & Irrégularité / imprévisibilité des horaires \\
$\checkmark$ & Evolution des rémunérations \\
Flexibilité temporelle ou financière
\end{tabular} & \begin{tabular}{ll} 
& \multicolumn{2}{c}{ Organisation du travail } \\
$\checkmark$ & Autonomie / contrainte \\
$\checkmark$ & «job enrichment » \\
$\checkmark$ & Polyvalence / polycompétence \\
$\checkmark$ & Délégation de responsabilité \\
$\checkmark$ & Travail d'équipe / équipes semi-autonomes \\
$\checkmark$ & Groupe de projet / travail en réseaux \\
$\checkmark$ & Coordination fonctionnelle \\
& Flexibilité fonctionnelle ou organisationnelle
\end{tabular} \\
\hline
\end{tabular}


Tableau 2 : Statistiques descriptives sur l'ancienneté dans l'entreprise et sur la stabilité de l'emploi Statistiques descriptives sur l'ensemble de la population

\begin{tabular}{|c|c|c|c|c|c|c|c|c|c|c|c|c|c|c|}
\hline & & \multicolumn{2}{|c|}{ Sexe } & \multirow[b]{2}{*}{ Totaux } & \multicolumn{4}{|c|}{ Age } & \multirow[b]{2}{*}{ Totaux } & \multicolumn{4}{|c|}{ Niveau d'études } & \multirow[b]{2}{*}{ Totaux } \\
\hline & & Homme & Femme & & $(15-24)$ ans & $(25-35)$ ans & (36-59) ans & $(60$ et +$)$ ans & & Aucun & Primaire & Secondaire & Supérieur & \\
\hline \multirow{2}{*}{$\begin{array}{l}\text { Ancienneté } \\
\text { dans } \\
\text { l'entreprise }\end{array}$} & $\begin{array}{c}\text { Inférieu } \\
\text { r ou égal } \\
\text { à } 2 \text { ans }\end{array}$ & $\begin{array}{c}622 \\
34.01 \%\end{array}$ & $\begin{array}{c}188 \\
41.78 \%\end{array}$ & $\begin{array}{c}810 \\
35.54 \%\end{array}$ & $\begin{array}{c}242 \\
63.68 \%\end{array}$ & $\begin{array}{c}387 \\
42.30 \%\end{array}$ & $\begin{array}{c}174 \\
18.59 \%\end{array}$ & $\begin{array}{c}7 \\
14.58 \%\end{array}$ & $\begin{array}{c}810 \\
35.54 \%\end{array}$ & $\begin{array}{c}244 \\
36.09 \%\end{array}$ & $\begin{array}{c}173 \\
38.27 \%\end{array}$ & $\begin{array}{c}309 \\
35.76 \%\end{array}$ & $\begin{array}{c}84 \\
29.27 \%\end{array}$ & $\begin{array}{c}810 \\
35.54 \%\end{array}$ \\
\hline & $\begin{array}{c}\text { Plus de } 2 \\
\text { ans }\end{array}$ & $\begin{array}{c}1207 \\
65.99 \%\end{array}$ & $\begin{array}{c}262 \\
58.22 \%\end{array}$ & $\begin{array}{c}1469 \\
64.46 \%\end{array}$ & $\begin{array}{c}138 \\
36.32 \%\end{array}$ & $\begin{array}{c}528 \\
57.70 \%\end{array}$ & $\begin{array}{c}762 \\
81.41 \%\end{array}$ & $\begin{array}{c}41 \\
85.42 \%\end{array}$ & $\begin{array}{c}1469 \\
64.46 \%\end{array}$ & $\begin{array}{c}432 \\
63.91 \%\end{array}$ & $\begin{array}{c}279 \\
61.73 \%\end{array}$ & $\begin{array}{c}555 \\
64.24 \%\end{array}$ & $\begin{array}{c}203 \\
70.73 \%\end{array}$ & $\begin{array}{c}1469 \\
64.46 \%\end{array}$ \\
\hline \multicolumn{2}{|c|}{ Totaux } & $\begin{array}{c}1829 \\
100 \%\end{array}$ & $\begin{array}{c}450 \\
100 \%\end{array}$ & $\begin{array}{c}2279 \\
100 \%\end{array}$ & $\begin{array}{c}380 \\
100 \%\end{array}$ & $\begin{array}{c}915 \\
100 \%\end{array}$ & $\begin{array}{c}936 \\
100 \%\end{array}$ & $\begin{array}{c}49 \\
100 \%\end{array}$ & $\begin{array}{c}2279 \\
100 \%\end{array}$ & $\begin{array}{c}676 \\
100 \%\end{array}$ & $\begin{array}{c}452 \\
100 \%\end{array}$ & $\begin{array}{c}864 \\
100 \%\end{array}$ & $\begin{array}{c}287 \\
100 \%\end{array}$ & $\begin{array}{c}2279 \\
100 \%\end{array}$ \\
\hline
\end{tabular}

\begin{tabular}{|c|c|c|c|c|c|c|c|c|c|c|c|}
\hline & \multicolumn{4}{|c|}{ Type de formation } & \multirow[b]{2}{*}{ Totaux } & \multicolumn{4}{|c|}{ Type d'entreprise } & \multirow[b]{2}{*}{ Totaux } \\
\hline & & $\begin{array}{c}\text { Autre } \\
\text { type }\end{array}$ & Général & Technique & Professionnel & & $\begin{array}{l}\text { Publique ou } \\
\text { parapublique }\end{array}$ & $\begin{array}{c}\text { Privée non } \\
\text { agricole }\end{array}$ & $\begin{array}{c}\text { Privée } \\
\text { agricole }\end{array}$ & $\begin{array}{c}\text { Autres } \\
\text { entreprises } \\
\end{array}$ & \\
\hline \multirow{2}{*}{$\begin{array}{c}\text { Ancienneté } \\
\text { dans } \\
\text { l'entreprise }\end{array}$} & $\begin{array}{l}\text { Inférieur ou } \\
\text { égal à } 2 \text { ans }\end{array}$ & $\begin{array}{c}252 \\
35.39 \% \\
\end{array}$ & $\begin{array}{c}499 \\
35.74 \% \\
\end{array}$ & $\begin{array}{c}37 \\
33.64 \% \\
\end{array}$ & $\begin{array}{c}22 \\
36.07 \% \\
\end{array}$ & $\begin{array}{c}810 \\
35.54 \% \\
\end{array}$ & $\begin{array}{c}85 \\
17.07 \% \\
\end{array}$ & $\begin{array}{c}557 \\
41.75 \% \\
\end{array}$ & $\begin{array}{c}160 \\
38.83 \% \\
\end{array}$ & $\begin{array}{c}8 \\
22.86 \% \\
\end{array}$ & $\begin{array}{c}810 \\
35.54 \% \\
\end{array}$ \\
\hline & Plus de 2 ans & $\begin{array}{c}460 \\
64.61 \% \\
\end{array}$ & $\begin{array}{c}897 \\
64.26 \% \\
\end{array}$ & $\begin{array}{c}73 \\
66.36 \% \\
\end{array}$ & $\begin{array}{c}39 \\
63.93 \% \\
\end{array}$ & $\begin{array}{c}1469 \\
64.46 \% \\
\end{array}$ & $\begin{array}{c}413 \\
92.93 \% \\
\end{array}$ & $\begin{array}{c}777 \\
58.25 \% \\
\end{array}$ & $\begin{array}{c}252 \\
61.17 \% \\
\end{array}$ & $\begin{array}{c}27 \\
77.14 \% \\
\end{array}$ & $\begin{array}{r}1469 \\
64.46 \% \\
\end{array}$ \\
\hline \multicolumn{2}{|c|}{ Totaux } & $\begin{array}{c}712 \\
100 \%\end{array}$ & $\begin{array}{l}1396 \\
100 \%\end{array}$ & $\begin{array}{c}110 \\
100 \%\end{array}$ & $\begin{array}{c}61 \\
100 \%\end{array}$ & $\begin{array}{l}2279 \\
100 \%\end{array}$ & $\begin{array}{c}498 \\
100 \%\end{array}$ & $\begin{array}{c}1334 \\
100 \%\end{array}$ & $\begin{array}{c}412 \\
100 \%\end{array}$ & $\begin{array}{c}35 \\
100 \%\end{array}$ & $\begin{array}{l}2279 \\
100 \%\end{array}$ \\
\hline
\end{tabular}

\begin{tabular}{|c|c|c|c|c|c|}
\hline & & \multicolumn{3}{|c|}{ Milieu de résidence } & \multirow[b]{2}{*}{ Totaux } \\
\hline & & Abidjan & $\begin{array}{c}\text { Autres } \\
\text { urbains }\end{array}$ & rural & \\
\hline \multirow{2}{*}{$\begin{array}{l}\text { Ancienneté dans } \\
\text { l'entreprise }\end{array}$} & $\begin{array}{l}\text { Inférieur ou } \\
\text { égal à } 2 \text { ans }\end{array}$ & $\begin{array}{c}219 \\
34.27 \%\end{array}$ & $\begin{array}{c}349 \\
34.93 \%\end{array}$ & $\begin{array}{c}242 \\
37.75 \%\end{array}$ & $\begin{array}{c}810 \\
35.54 \%\end{array}$ \\
\hline & Plus de 2 ans & $\begin{array}{c}420 \\
65.73 \%\end{array}$ & $\begin{array}{c}650 \\
65.07 \%\end{array}$ & $\begin{array}{c}399 \\
62.25 \%\end{array}$ & $\begin{array}{c}1469 \\
64.46 \%\end{array}$ \\
\hline \multicolumn{2}{|c|}{ Totaux } & $\begin{array}{c}639 \\
100 \%\end{array}$ & $\begin{array}{c}999 \\
100 \%\end{array}$ & $\begin{array}{c}641 \\
100 \%\end{array}$ & $\begin{array}{l}2279 \\
100 \%\end{array}$ \\
\hline
\end{tabular}




\section{$\underline{\text { Statistiques descriptives sur la sous-population des travailleurs occupant un emploi stable }}$}

\begin{tabular}{|c|c|c|c|c|c|c|c|c|c|c|c|c|c|c|}
\hline & \multicolumn{2}{|c|}{ Sexe } & \multirow[b]{2}{*}{ Totaux } & \multicolumn{4}{|c|}{ Situation matrimoniale } & \multirow[b]{2}{*}{ Totaux } & \multicolumn{4}{|c|}{ Niveau d'études } & \multirow[t]{2}{*}{ Totaux } \\
\hline & & Homme & Femme & & Marié & $\begin{array}{l}\text { Divorcé/ } \\
\text { Séparé }\end{array}$ & $\begin{array}{l}\text { Veuf/ } \\
\text { Veuve }\end{array}$ & $\begin{array}{c}\text { Jamais } \\
\text { marié/ } \\
\text { Célibataire }\end{array}$ & & Aucun & Primaire & Secondaire & Supérieur & \\
\hline \multirow{2}{*}{$\begin{array}{c}\text { Emploi } \\
\text { stable }\end{array}$} & Non & $\begin{array}{c}814 \\
67.44 \%\end{array}$ & $\begin{array}{c}142 \\
56.87 \%\end{array}$ & $\begin{array}{c}963 \\
65.55 \%\end{array}$ & $\begin{array}{c}467 \\
57.58 \%\end{array}$ & $\begin{array}{c}25 \\
80.65 \%\end{array}$ & $\begin{array}{c}11 \\
52.38 \%\end{array}$ & $\begin{array}{c}460 \\
75.91 \%\end{array}$ & $\begin{array}{c}963 \\
65.55 \%\end{array}$ & $\begin{array}{c}393 \\
90.97 \%\end{array}$ & $\begin{array}{c}241 \\
86.38 \%\end{array}$ & $\begin{array}{c}282 \\
50.81 \%\end{array}$ & $\begin{array}{c}47 \\
23.15 \%\end{array}$ & $\begin{array}{c}963 \\
65.55 \%\end{array}$ \\
\hline & Oui & $\begin{array}{c}393 \\
32.56 \%\end{array}$ & $\begin{array}{c}113 \\
43.13 \%\end{array}$ & $\begin{array}{c}506 \\
34.45 \%\end{array}$ & $\begin{array}{c}344 \\
42.42 \%\end{array}$ & $\begin{array}{c}6 \\
19.35 \%\end{array}$ & $\begin{array}{c}10 \\
47.62 \%\end{array}$ & $\begin{array}{c}146 \\
24.09 \%\end{array}$ & $\begin{array}{c}506 \\
34.45 \%\end{array}$ & $\begin{array}{c}39 \\
9.03 \%\end{array}$ & $\begin{array}{c}38 \\
13.62 \%\end{array}$ & $\begin{array}{c}273 \\
49.19 \%\end{array}$ & $\begin{array}{c}156 \\
76.85 \%\end{array}$ & $\begin{array}{c}506 \\
34.45 \%\end{array}$ \\
\hline \multicolumn{2}{|c|}{ Totaux } & $\begin{array}{c}1207 \\
100 \%\end{array}$ & $\begin{array}{c}262 \\
100 \%\end{array}$ & $\begin{array}{c}1469 \\
100 \%\end{array}$ & $\begin{array}{c}811 \\
100 \%\end{array}$ & $\begin{array}{c}31 \\
100 \%\end{array}$ & $\begin{array}{c}21 \\
100 \%\end{array}$ & $\begin{array}{c}606 \\
100 \%\end{array}$ & $\begin{array}{c}1469 \\
100 \%\end{array}$ & $\begin{array}{c}432 \\
100 \%\end{array}$ & $\begin{array}{c}279 \\
100 \%\end{array}$ & $\begin{array}{c}555 \\
100 \%\end{array}$ & $\begin{array}{c}203 \\
100 \%\end{array}$ & $\begin{array}{c}1469 \\
100 \%\end{array}$ \\
\hline
\end{tabular}

\begin{tabular}{|c|c|c|c|c|c|c|c|c|c|c|c|c|c|}
\hline & & \multicolumn{3}{|c|}{$\begin{array}{c}\text { Connaissances d'une autre } \\
\text { langue }\end{array}$} & \multirow[b]{2}{*}{ Totaux } & \multicolumn{3}{|c|}{ Connaissance de l'informatique } & \multirow[b]{2}{*}{ Totaux } & \multicolumn{3}{|c|}{ Expérience professionnelle } & \multirow[b]{2}{*}{ Totaux } \\
\hline & & Non & Lecture & $\begin{array}{c}\text { Lecture } \\
\text { et } \\
\text { écriture }\end{array}$ & & $\begin{array}{c}\text { Pas du } \\
\text { tout }\end{array}$ & Oui bon & $\begin{array}{c}\text { Oui } \\
\text { moyen }\end{array}$ & & $(0-2)$ ans & $(3-10)$ ans & $(11$ ans et +$)$ & \\
\hline \multirow{2}{*}{$\begin{array}{c}\text { Emploi } \\
\text { stable }\end{array}$} & Non & $\begin{array}{c}717 \\
78.88 \% \\
\end{array}$ & $\begin{array}{c}42 \\
56 \%\end{array}$ & $\begin{array}{c}204 \\
42.06 \% \\
\end{array}$ & $\begin{array}{c}963 \\
65.55 \% \\
\end{array}$ & $\begin{array}{c}761 \\
80.36 \%\end{array}$ & $\begin{array}{c}38 \\
27.54 \% \\
\end{array}$ & $\begin{array}{c}164 \\
42.71 \% \\
\end{array}$ & $\begin{array}{c}963 \\
65.55 \% \\
\end{array}$ & $\begin{array}{c}50 \\
72.46 \% \\
\end{array}$ & $\begin{array}{c}627 \\
70.93 \% \\
\end{array}$ & $\begin{array}{c}286 \\
55.43 \%\end{array}$ & $\begin{array}{c}963 \\
65.55 \% \\
\end{array}$ \\
\hline & Oui & $\begin{array}{c}192 \\
21.12 \%\end{array}$ & $\begin{array}{c}33 \\
44 \%\end{array}$ & $\begin{array}{c}281 \\
57.94 \%\end{array}$ & $\begin{array}{c}506 \\
34.45 \%\end{array}$ & $\begin{array}{c}186 \\
19.64 \%\end{array}$ & $\begin{array}{c}100 \\
72.46 \%\end{array}$ & $\begin{array}{c}220 \\
57.29 \%\end{array}$ & $\begin{array}{c}506 \\
34.45 \%\end{array}$ & $\begin{array}{c}19 \\
27.54 \%\end{array}$ & $\begin{array}{c}257 \\
29.07 \%\end{array}$ & $\begin{array}{c}230 \\
44.57 \%\end{array}$ & $\begin{array}{c}506 \\
34.45 \%\end{array}$ \\
\hline \multicolumn{2}{|c|}{ Totaux } & $\begin{array}{c}909 \\
100 \%\end{array}$ & $\begin{array}{c}75 \\
100 \%\end{array}$ & $\begin{array}{c}485 \\
100 \%\end{array}$ & $\begin{array}{l}1469 \\
100 \%\end{array}$ & $\begin{array}{c}947 \\
100 \%\end{array}$ & $\begin{array}{c}138 \\
100 \%\end{array}$ & $\begin{array}{c}384 \\
100 \%\end{array}$ & $\begin{array}{l}1469 \\
100 \%\end{array}$ & $\begin{array}{c}69 \\
100 \%\end{array}$ & $\begin{array}{c}884 \\
100 \%\end{array}$ & $\begin{array}{c}516 \\
100 \%\end{array}$ & $\begin{array}{l}1469 \\
100 \%\end{array}$ \\
\hline
\end{tabular}




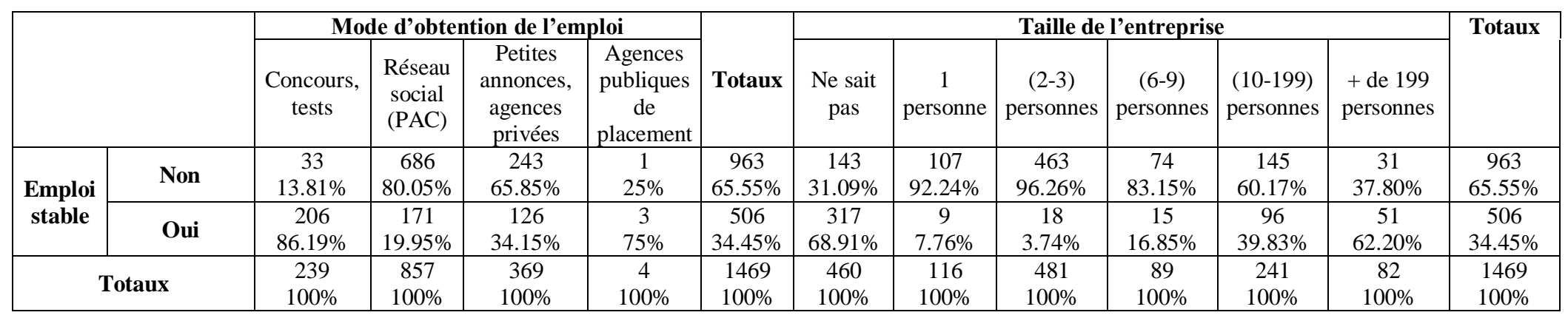

\begin{tabular}{|c|c|c|c|c|c|c|c|c|c|}
\hline & & \multicolumn{4}{|c|}{ Branche d'activité } & \multirow{2}{*}{ Totaux } & \multicolumn{2}{|c|}{$\begin{array}{c}\text { Existence de syndicat en } \\
\text { entreprise }\end{array}$} & \multirow{2}{*}{ Totaux } \\
\hline & & Agriculture & Industrie & Commerce & Service & & Oui & Non & \\
\hline \multirow{2}{*}{ Emploi stable } & Non & $\begin{array}{c}263 \\
92.61 \%\end{array}$ & $\begin{array}{c}126 \\
69.61 \%\end{array}$ & $\begin{array}{c}159 \\
81.12 \%\end{array}$ & $\begin{array}{c}415 \\
51.36 \%\end{array}$ & $\begin{array}{c}963 \\
65.55 \%\end{array}$ & $\begin{array}{c}124 \\
27.62 \%\end{array}$ & $\begin{array}{c}832 \\
82.25 \%\end{array}$ & $\begin{array}{c}963 \\
65.55 \%\end{array}$ \\
\hline & Oui & $\begin{array}{c}21 \\
7.39 \%\end{array}$ & $\begin{array}{c}55 \\
30.39 \%\end{array}$ & $\begin{array}{c}37 \\
18.88 \%\end{array}$ & $\begin{array}{c}393 \\
48.64 \%\end{array}$ & $\begin{array}{c}506 \\
34.45 \%\end{array}$ & $\begin{array}{c}325 \\
72.38 \%\end{array}$ & $\begin{array}{c}181 \\
17.75 \%\end{array}$ & $\begin{array}{c}506 \\
34.45 \%\end{array}$ \\
\hline \multicolumn{2}{|c|}{ Totaux } & $\begin{array}{c}284 \\
100 \%\end{array}$ & $\begin{array}{c}181 \\
100 \%\end{array}$ & $\begin{array}{c}196 \\
100 \%\end{array}$ & $\begin{array}{c}808 \\
100 \%\end{array}$ & $\begin{array}{l}1469 \\
100 \%\end{array}$ & $\begin{array}{c}449 \\
100 \%\end{array}$ & $\begin{array}{l}1020 \\
100 \%\end{array}$ & $\begin{array}{l}1469 \\
100 \%\end{array}$ \\
\hline
\end{tabular}

Source : calculs de l'auteur à partir de l'ENSESI 2016.

Tableau 3 : Probit binaire avec sélection par la méthode du maximum de vraisemblance

\begin{tabular}{l}
\hline Equation substant \\
\hline Variables \\
\\
Sexe [Réf. homme] \\
Femme
\end{tabular}

Femme

Coefficients $\begin{aligned} & \text { Ecarts- } \\ & \text { type }\end{aligned}$

$.1886^{*} \quad .0972$

\begin{tabular}{ll}
\multicolumn{2}{c}{ Effets marginaux } \\
Coefficients & $\begin{array}{l}\text { Ecarts- } \\
\text { type }\end{array}$ \\
$.0742 * *$ & .0385
\end{tabular}

\begin{tabular}{||l} 
Equation de sélection \\
Variables \\
Sexe [Réf. homme] \\
Femme
\end{tabular}

\section{Coefficients Ecarts-type}

$-.2203 * * * \quad .0712$ 
Situation matrimoniale [Réf. marié] Divorcé / Séparé

Veuf / Veuve

Jamais marié / Célibataire

Niveau d'études [Réf. aucun]

Primaire

Secondaire

Supérieur

Connaissances d'une autre langue

\section{[Réf. non]}

Lecture uniquement

Lecture et écriture

Connaissance de l'informatique [Réf. pas du tout]

Oui bon

Oui moyen

$.3360 * *$

.1320

.1338

.0930

$.1330 * *$

.0517

.0530

Expérience professionnelle [Réf. (0-2) ans]

(3-10) ans

.1117

11 ans et +

Mode d'obtention de l'emploi [Réf. concours]

Parents-Amis-Connaissances (PAC)

Petites annonces ou annonces privées FNJ, AGEPE

$\begin{array}{llll}-.4005 * * * & .1363 & -.1511 * * * & .0497\end{array}$

$\begin{array}{llll}.0081 & .6601 & .0032 & .2577\end{array}$

\section{Age [Réf. (15-24) ans]}

$(25-35)$ ans

(36-59) ans

$.5352 * * *$

$1.1459 * * *$

$1.4247 * * * \quad .2166$

60 ans et +

Niveau d'études [Réf. aucun]

Primaire

Secondaire

.3233

.1728

.0561

.2161

Supérieur

.2215
.2382

Type de formation [Réf. autres

types]

Général

Technique

Professionnel

$-.3638 *$

$-.2381$

$-.4873 *$

2125

.2469

.2673

Type d'entreprise [Réf. admin.

Publique]

Entreprise privée non agricole

Entreprise agricole

Autres entreprises (ONG,

Association)

$-.6346 * * *$

$-.5018 * * * \quad .0837$

$5051 * * \quad .1132$

Milieu de résidence [Réf.

Abidjan]

$-.1130 *$

$-.1130 *$
-.1037

.0652

Autres urbains

Rural

$.4099 * * *$

Constante 


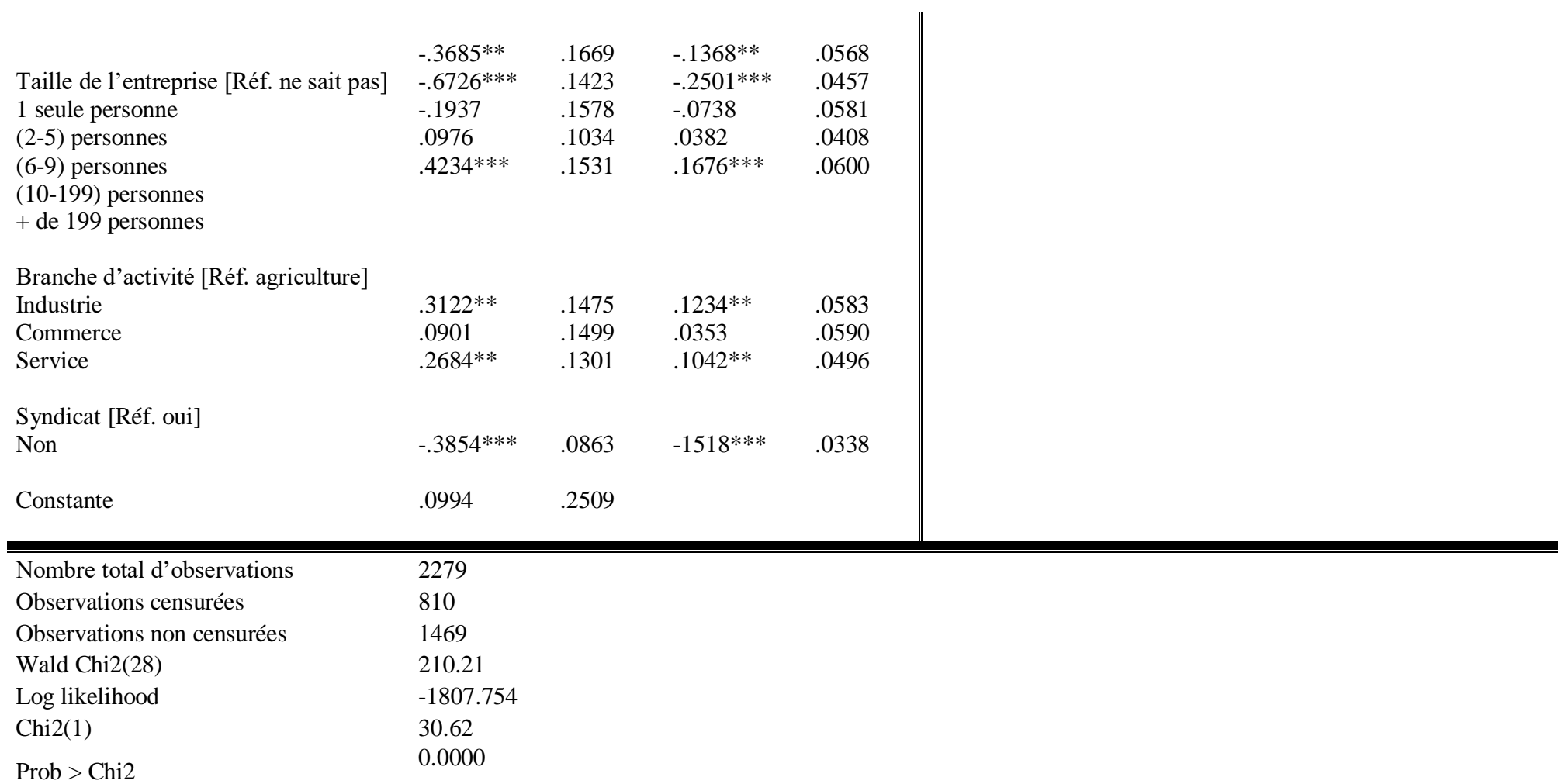

$* ; * ; * * *$ : significativités respectives au seuil de $10 \% ; 5 \%$ et $1 \%$

Source : calculs de l'auteur à partir de l'ENSESI 2016. 